\title{
Exercício aeróbico e intensidade autosselecionada por mulheres: uma revisão sistemática
}

\section{Aerobic exercise and self-selected intensity by women: a systematic review}

\author{
Flávia Xavier de Andrade Lage, Paulo Roberto dos Santos Amorim, \\ Osvaldo Costa Moreira, Renata Aparecida Rodrigues de Oliveira, \\ João Carlos Bouzas Marins
}

Como citar este artigo: LACE, FLÁVIA X. A.; AMORIM,

PAULO R. S.; MOREIRA, OSVALDO C.; OLIVEIRA, RENATA A. R.; MARINS, JOÃO C. B.

Exercício aeróbico e intensidade autosselecionada por mulheres: uma revisão sistemática. Revista Saúde (Sta. Maria). 2020; 46 (2).

\section{Autor correspondente:}

Nome: Flávia Xavier de Andrade Lage

E-mail: flaviaxavier.lage@yahoo.com.br Telefone: (31) 3899-2076

Formação Profissional: Mestre em Educacão Física pela (o) Universidade Federal de Viçosa (UFV) que fica na cidade de Viçosa, Minas Gerais, Brasil.

Filiação Institucional: Universidade Federal de Viçosa

Endereço para correspondência: Avenida Peter Henry Rolfs, s/n

Campus Universitário

Cidade: Vicosa

Estado: Minas Gerais

CEP: 36570-900

Data de Submissão:

09/05/2020

Data de aceite:

27/08/2020

Conflito de Interesse: Não há conflito de interesse

\section{(cc) $\mathrm{BY}-\mathrm{NC}-\mathrm{ND}$}

\section{RESUMO}

Objetivo: Identificar o perfil das praticantes de exercícios aeróbicos sob intensidade autosselecionada. Métodos: Analisou-se os dados a partir das bases de dados PubMed e BVS, com artigos originais, realizados em mulheres acima de 18 anos de idade, publicados nos últimos 10 anos, com análise de exercício aeróbico de forma autosselecionada através da mensuração da frequência cardíaca (FC) e percepção subjetiva do esforço (PSE), sendo incluídos 20 artigos. Resultados: Foi encontrado em 15 estudos que a intensidade autosselecionada estaria dentro das recomendações para melhorar a saúde, além de obter resposta afetiva positiva e maior prazer quando comparado à intensidade imposta. Pessoas com sobrepeso e obesidade demonstraram um menor gasto energético quando comparado com pessoas eutróficas, além de caminharem em velocidade inferior. A idade parece não influenciar nas respostas afetivas, apenas nas respostas fisiológicas. Foi encontrado também maior sensação de prazer ao realizar atividade física (AF) ao ar livre quando comparado com ambiente interno. Conclusão: Quando a intensidade do exercício é autosselecionada, há maiores sensações de prazer e atendem às recomendações do American College of Sports Medicine (ACSM), além de promover melhorias na aptidão física.

PALAVRAS-CHAVE: Exercício aeróbico, Autosselecionado, Frequência cardíaca.

\section{ABSTRACT}

Objective: Identify the profile of practitioners of aerobic exercises under self-selected intensity. Methods: Data were analysed from PubMed and BVS databases, with original articles, performed in women who were above 18 years old, published in last 10 years, with analysis of self-selected aerobic exercise through the heart rate (HR) measuring, being included 20 articles. Results: It was found in 15 studies that the self-selected intensity would be within the recommendations to improve health, the affective response was positive and, when compared to the imposed intensity, more pleasure was felt in exercise. Individuals with overweight or obesity showed a lower energy expenditure than eutrophic persons, and also used to walk in lower speeds. Age doesn't seem to have an effect on the affective responses, but only in the physiologic ones. Also, was found more pleasure feeling when doing PA outdoors, in comparison with indoor environments. Conclusion: When the exercise intensity is selfselected, there are more pleasure feelings and the ACSM recommendations are meet, besides to help to improve the physical fitness.

KEYWORDS: Aerobic exercise, Self-selected, Heart rate. 


\section{INTRODUÇÃO}

Sobrepeso e obesidade têm sido um dos maiores problemas de saúde em nível global, incluindo o Brasil, correspondendo a valores de $53,8 \%$ e $18,9 \%$ respectivamente ${ }^{1}$. Um dos principais fatores que contribuem para esta elevada taxa de sobrepeso e obesidade está relacionado ao estilo de vida, com adoção de comportamentos sedentários, com prevalência entre $44,4 \%$ e $56,0 \%$ nas regiões brasileiras ${ }^{2}$.

A prática de atividades físicas no tempo livre, no Brasil, gira em torno de $46,6 \%$ para os homens e $29,9 \%$ para as mulheres ${ }^{1}$. Especificamente 0 comportamento adotado por mulheres indica maior taxa de inatividade física em comparação aos homens. Todos esses dados reforçam que a maior parte da população brasileira possui hábitos sedentários ${ }^{3}$. Cabe destacar que os impactos positivos à saúde relacionados a atividade física são condicionados ao alcance de níveis mínimos, capazes de induzir a alterações fisiológicas importantes ${ }^{4}$.

De acordo com o American College of Sports Medicine (ACSM) ${ }^{4}$, o gasto energético de aproximadamente 1000 quilocalorias (kcal) por semana ou acúmulo de aproximadamente 150 minutos por semana de atividade física de intensidade moderada está associado a baixas taxas de risco cardiovascular e obtenção de melhorias na capacidade cardiorrespiratória. A caminhada é a atividade física mais praticada no tempo livre entre os brasileiros ${ }^{5}$, sendo comumente recomendada devido a fácil execução ${ }^{6}$, baixo custo, além de ser uma atividade natural inerente à vida cotidiana, o que acaba levando o praticante a exercer, uma autosseleção de sua intensidade baseada no próprio conforto ${ }^{7}$.

A autosseleção se baseia na escolha do próprio praticante de um ritmo, velocidade ou intensidade de sua preferência ${ }^{7}$. Entretanto, o ACSM4 recomenda que, para realizar um adequado controle de intensidade de treinamento, é importante atingir valores entre 60 a $90 \%$ da frequência cardíaca máxima $\left(\mathrm{FC}_{\text {máx }}\right)$, ou 50 a $85 \%$ do consumo máximo de oxigênio $\left(\mathrm{VO}_{2 \text { máx }}\right)^{4}$. Um aspecto importante compreende os fatores que influenciam na aderência ao exercício físico. A intensidade com que ele é realizado representa um fator que influencia a taxa de adesão, que é substancialmente menor quando ocorre em intensidades mais elevadas, segundo o $\mathrm{ACSM}^{4}$. Existem várias formas de se quantificar a intensidade de um exercício. Dentre as medidas mais habituais, tem-se a frequência cardíaca (FC) e a escala de Borg ${ }^{8}$. A FC estabelece indicadores objetivos da resposta cardiovascular durante o exercício $0^{4,6,9}$, enquanto a escala de Borg ${ }^{8}$ possui um enfoque psicofisiológico. Alguns estudos relacionados à avaliação da autosseleção de intensidades de exercícios físicos já foram relatados em diversos grupos, faixas etárias e tipos de exercícios aeróbicos ${ }^{6,10,11}$. Apesar de evidências apontarem que os indivíduos são capazes de autosselecionar uma intensidade confortável e adequada para promover benefícios cardiorrespiratórios, outras apontam que tal intensidade pode ser elevada para pessoas com sobrepeso e obesidade, por exemplo?.

Um adequado controle da intensidade do exercício, com a intensidade dentro dos padrões recomendados, é importante para, principalmente, obter melhorias no sistema cardiorrespiratório, para diminuição de gordura corporal, redução dos riscos para doenças cardiovasculares, variando de acordo com o condicionamento e objetivos do praticante 4 . 
Desta forma, é interessante estabelecer se sujeitos que realizam um exercício com intensidade autosselecionada, acabam escolhendo a faixa de intensidade adequada, segundo as orientações para prescrição de exercícios ${ }^{4}$.

Tendo em vista que a maior parte da população realiza exercício físico de forma não supervisionada, é interessante realizar um levantamento sobre o tema, para identificar a adequabilidade da autosseleção das intensidades. Tomando como referência um levantamento nas bases de dados PubMed e Biblioteca Virtual em Saúde (BVS/Bireme), feito em 26/03/2018 com as palavras-chaves em inglês "exercise"AND "self-selected"AND "heart rate", foi possível observar que ainda não há um consenso sobre a eficácia do exercício aeróbico realizado sob intensidade autosselecionada.

Estudos demonstraram que homens e mulheres tendem a selecionar velocidades diferentes ${ }^{12} \mathrm{e}$, devido às diferenças morfofisiológicas entre os sexos, a intensidade e solicitação de substratos durante o exercício, as intensidades e gasto energético (GE) tendem a ser diferentes, reforçando a importância de se conhecer especificamente o comportamento autosselecionado de exercícios em mulheres. Portanto, esta revisão sistemática será conduzida com o objetivo geral de identificar o perfil das praticantes de exercícios aeróbicos sob intensidade autosselecionada e, de forma específica, verificar se fatores antropométricos, fisiológicos, ambientais e idade influenciam na intensidade autosselecionada, bem como no aumento da adesão de exercícios aeróbicos.

\section{MÉTODOS}

\section{Estratégias de Pesquisa}

Para realizar a presente revisão sistemática, foi feita uma busca nas bases de dados PubMed e Biblioteca Virtual em Saúde (BVS/Bireme), utilizando os seguintes termos: "exercise" AND "self-selected" AND "heart rate". Na base de dados PubMed e BVS, utilizou-se como filtros de busca artigos originais publicados nos últimos 10 anos (Janeiro de 2008 a Março de 2018), realizados com seres humanos do sexo feminino e com faixa etária acima de 18 anos.

\section{Critérios de Inclusão}

Para que os artigos fossem incluídos no estudo, era necessário que englobassem os seguintes aspectos: 1) ser artigo original; 2) estudos que contenham análise de exercício aeróbico de forma autosselecionada através da mensuração da FC e ou percepção subjetiva de esforço (PSE); 3) mulheres acima de 18 anos; 4) artigos publicados em Inglês, Português ou Espanhol; 5) publicações nos últimos 10 anos (janeiro de 2008 até março de 2018).

Todos os artigos encontrados no processo de busca foram inicialmente analisados pelo título e resumo para verificar se atendiam aos objetivos do estudo e aos critérios de inclusão e exclusão. Caso o título e ou resumo não apresentassem clareza, realizava-se uma leitura na metodologia no corpo do artigo para confirmar se estava de acordo 
com o propósito do presente estudo. Em casos de discordância entre os avaliadores, foi solicitada uma terceira opinião de outro avaliador.

Destaca-se que os artigos deveriam responder tais perguntas: 1) Quais as características dos praticantes de exercícios aeróbicos que utilizam a intensidade autosselecionada e quais as formas de registro da intensidade estão presentes? 2)Quais os resultados já encontrados em diferentes tipos de grupo? 3)Quais fatores (antropométricos, fisiológicos, ambientais, gênero, idade) parecem influenciar na intensidade autosselecionada? 4) A intensidade autosselecionada influencia no aumento da aderência da prática de exercícios aeróbicos?

Foi realizada a análise da qualidade metodológica dos estudos através da escala de Downs e Black ${ }^{13}$ adaptada para a inclusão de estudos transversais ${ }^{14}$. Tal escala avalia os estudos através de 17 questões, englobando a validade externa, validade interna e as informações fornecidas no artigo, atribuindo-se um valor máximo de 18 pontos. Para extração e registro dos dados dos estudos analisados, foi utilizado Microsoft Office Excel 2010.

\section{RESULTADOS}

A Figura 1 ilustra o processo de seleção dos artigos para compor esta revisão sistemática.

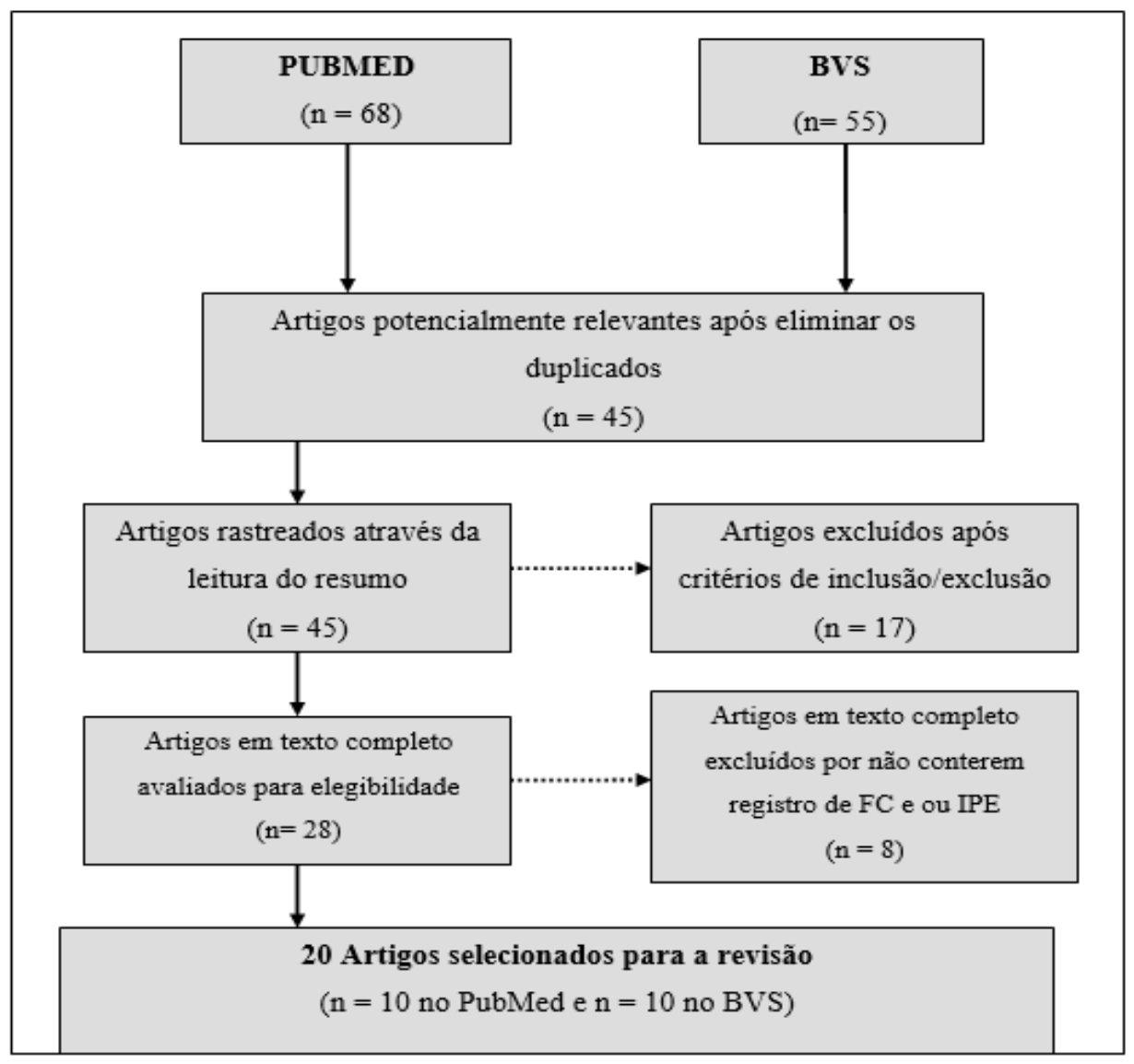

Figura 1. Fluxograma do processo de seleção dos artigos. 
Na presente revisão sistemática foram considerados os estudos que atingiram, pelo menos, $50 \%$ do escore de qualidade metodológica, segundo a escala de Downs e Black ${ }^{13}$ adaptada para a inclusão de estudos transversais ${ }^{14}$. A Tabela 1 apresenta os estudos incluídos na presente revisão e com o escore obtido.

Tabela 1. Análise da qualidade metodológica dos estudos incluídos na revisão

\begin{tabular}{lc}
\hline Estudo & Escore - Frequência (\%) \\
\hline Elsangedy et al. $^{9}$ & $9(50)$ \\
Krinski et al. $^{15}$ & $9(50)$ \\
Glen et al. $^{10}$ & $11(61,1)$ \\
Sherman et al. $^{16}$ & $10(55,6)$ \\
Lattari et al. $^{17}$ & $11(61,1)$ \\
Peterman et al. ${ }^{18}$ & $11(61,1)$ \\
Zdziarski et al. $^{19}$ & $10(55,6)$ \\
Smith et al. $^{20}$ & $12(66,7)$ \\
Almeida et al. ${ }^{21}$ & $9(50)$ \\
Orepic et al. ${ }^{22}$ & $10(55,6)$ \\
Douris et al. ${ }^{23}$ & $10(55,6)$ \\
DaSilva et al. ${ }^{24}$ & $10(55,6)$ \\
Simpson; Munro; Steele ${ }^{25}$ & $9(50)$ \\
DaSilva et al. & \\
Buzzachera et al. ${ }^{26}$ & $9(50)$ \\
DaSilva et al. ${ }^{27}$ & $9(50)$ \\
Elsangedy et al. ${ }^{28}$ & $10(55,6)$ \\
Hansen; Smith ${ }^{11}$ & $9(50)$ \\
Rendi et al. ${ }^{29}$ & $11(61,1)$ \\
DaSilva et al. ${ }^{3}$ & $9(50)$ \\
\hline
\end{tabular}

A Tabela 2 apresenta uma síntese dos trabalhos considerados neste estudo, indicando o objetivo, características da amostra, idade, tipo de exercício realizado, forma de registro dos dados e principais resultados encontrados 
Tabela 2. Características gerais dos estudos incluídos.

\begin{tabular}{|c|c|c|c|c|c|c|}
\hline Referência & Objetivo & Amostra & Idade & $\begin{array}{c}\text { Tipo de } \\
\text { exercício }\end{array}$ & $\begin{array}{c}\text { Forma de } \\
\text { registro }\end{array}$ & Resultado \\
\hline ELSANGEDY et al. ${ }^{9}$ & $\begin{array}{l}\text { Verificar a associação entre o IMC e } \\
\text { as respostas afetivas, avaliações do } \\
\text { IPE e a demanda fisiológica durante o } \\
\text { exercício individual. }\end{array}$ & $\begin{array}{c}\mathrm{G} 1=22 \\
\text { mulheres } \\
\text { eutróficas } \\
\mathrm{G} 2=22 \\
\text { mulheres } \\
\text { sobrepeso } \\
\mathrm{G} 3=22 \\
\text { mulheres } \\
\text { obesas }\end{array}$ & 33,1 anos & Esteira & $\begin{array}{c}\mathrm{FC}, \mathrm{VO}_{2} \text { máx } \\
\mathrm{IPE}\end{array}$ & $\begin{array}{l}\text { A obesidade está associada a uma diminuição } \\
\text { da resposta afetiva para a mesma intensidade } \\
\text { de exercício autosselecionado (AS). Uma } \\
\text { análise de regressão múltipla mostrou que } \\
\text { tanto o IMC quanto o IPE foram preditores } \\
\text { significativos de resposta afetiva durante } \\
\text { o exercício individual, enquanto nenhuma } \\
\text { resposta fisiológica predisse resposta afetiva. } \\
\text { Além disso, a intensidade do exercício de } \\
\text { caminhada em ritmo AS em mulheres com IMC } \\
\text { diferente gera efeito positivo e está dentro da } \\
\text { faixa recomendada para melhorar a saúde. }\end{array}$ \\
\hline KRINSKI et al. ${ }^{15}$ & $\begin{array}{l}\text { Comparar a influência de uma única } \\
\text { sessão de caminhada em ritmo AS em } \\
\text { ambiente interno e externo sobre as } \\
\text { respostas fisiológicas e psicológicas } \\
\text { em mulheres obesas. }\end{array}$ & $\begin{array}{c}38 \text { mulheres } \\
\text { obesas }\end{array}$ & $\begin{array}{l}45,64 \\
\text { anos }\end{array}$ & Esteira e pista & $\begin{array}{l}\mathrm{FC}, \mathrm{VO}_{2} \text { máx } \\
\text { IPE, VA, EA, } \\
\text { FAS }\end{array}$ & $\begin{array}{c}\text { Em relação às respostas fisiológicas, } \\
\text { as participantes autosselecionaram } \\
\text { intensidades suficientes para a melhoria do } \\
\text { condicionamento físico nos dois ambientes. } \\
\text { Entretanto, as avaliadas apresentaram maior } \\
\text { prazer ao realizar AF ao ar livre. }\end{array}$ \\
\hline GLEN et al. ${ }^{10}$ & $\begin{array}{l}\text { Comparar a intensidade de um } \\
\text { exercício com intensidade AS em } \\
\text { cicloergômetro com e sem modo de } \\
\text { jogo ativados (exergaming) }\end{array}$ & $\begin{array}{l}20 \text { homens } \\
18 \text { mulheres }\end{array}$ & $\begin{array}{l}30,9 \text { anos } \\
30,9 \text { anos }\end{array}$ & Cicloergômetro & $\begin{array}{l}\text { Calorimetria } \\
\text { indireta, GE, } \\
\text { FC, IPE, EA }\end{array}$ & $\begin{array}{l}\text { Os indivíduos autosselecionaram intensidade } \\
\text { próxima ao LV, relatando resposta afetiva } \\
\text { positiva. Com o modo de jogo ativado, } \\
\text { obtiveram maior intensidade e maior prazer. }\end{array}$ \\
\hline SHERMAN et al. ${ }^{16}$ & $\begin{array}{c}\text { Comparar intensidade através da } \\
\text { FC entre caminhada com ritmo } \\
\text { AS e caminhada com intensidade } \\
\text { equivalente a de uma sessão de Yoga. }\end{array}$ & 16 mulheres & $\begin{array}{l}24,15 \\
\text { anos }\end{array}$ & Esteira & $\begin{array}{l}\text { FC, IPE, VA, } \\
\text { EA, PACES }\end{array}$ & $\begin{array}{l}\text { A caminhada com intensidade equivalente a } \\
\text { de uma sessão de Yoga foi significativamente } \\
\text { menor do que a realizada em intensidade AS. }\end{array}$ \\
\hline
\end{tabular}




\begin{tabular}{|c|c|c|c|c|c|c|}
\hline LATTARI et al. ${ }^{17}$ & $\begin{array}{c}\text { Comparar as respostas afetivas e a } \\
\text { assimetria frontal do EEG induzida por } \\
\text { sessões de exercícios prescritos e de } \\
\text { intensidade AS. }\end{array}$ & $\begin{array}{l}20 \text { mulheres } \\
\text { saudáveis }\end{array}$ & 26,5 anos & Cicloergômetro & $\begin{array}{l}\mathrm{FC}, \mathrm{VO}_{2} \text { máx, } \\
\text { IPE,VA, FAS }\end{array}$ & $\begin{array}{l}\text { Embora não tenha ocorrido assimetria frontal } \\
\text { após a condição de exercício com intensidade } \\
\text { AS, esta apresentou eficácia para induzir } \\
\text { mudanças positivas no estado afetivo. }\end{array}$ \\
\hline PETERMAN et al. ${ }^{18}$ & $\begin{array}{l}\text { Verificar a AF AS realizada por } \\
\text { voluntários adeptos ao pedelec e } \\
\text { examinar como o deslocamento } \\
\text { diário por } 4 \text { semanas pode influenciar } \\
\text { fatores de risco cardiometabólico. }\end{array}$ & $\begin{array}{l}6 \text { homens } \\
14 \text { mulheres }\end{array}$ & 41,5 anos & Pedelec & $\begin{array}{l}\text { FC, GPS, } \\
\text { distância, } \\
\text { tempo total }\end{array}$ & $\begin{array}{c}\text { Os participantes autosselecionaram uma } \\
\text { intensidade moderada para pedalar e } \\
\text { foram associados a melhorias significativas } \\
\text { em alguns dos principais fatores de risco } \\
\text { cardiometabólico. }\end{array}$ \\
\hline ZDZIARSKI et al. ${ }^{19}$ & $\begin{array}{c}\text { Determinar as diferenças } \\
\text { das respostas cinemáticas, } \\
\text { cardiopulmonares e metabólicas } \\
\text { entre corredores acima do peso e } \\
\text { saudáveis, a uma intensidade AS. }\end{array}$ & $\begin{array}{l}21 \text { sobrepeso } \\
42 \text { eutróficas }\end{array}$ & $\begin{array}{l}41,9 \text { anos } \\
43,6 \text { anos }\end{array}$ & Esteira & $\begin{array}{c}\mathrm{FC}, \mathrm{VO}_{2} \text { máx, } \\
\mathrm{GE}\end{array}$ & $\begin{array}{l}\text { Corredores com sobrepeso demonstraram } \\
\text { GE significativamente menor quando } \\
\text { autosselecionaram a intensidade, que pode } \\
\text { não ser metabolicamente adequado para } \\
\text { perda de peso e saúde. }\end{array}$ \\
\hline SMITH et al. ${ }^{20}$ & $\begin{array}{c}\text { Examinar o padrão de respostas } \\
\text { fisiológicas e psicológicas em adultos } \\
\text { mais velhos durante teste de exaustão } \\
\text { e uma sessão de } 20^{\prime} \text { de exercício com } \\
\text { intensidade AS. }\end{array}$ & $\begin{array}{l}10 \text { homens } \\
8 \text { mulheres }\end{array}$ & $\begin{array}{c}65 \text { anos } \\
63,7 \text { anos }\end{array}$ & Esteira & $\begin{array}{l}\mathrm{FC}, \mathrm{IPE} \\
\mathrm{VO}_{2} \max \end{array}$ & $\begin{array}{l}\text { Evidenciou estabilidade relativa do } \\
\text { efeito positivo, próximo ao LV quando } \\
\text { autosselecionaram a intensidade. }\end{array}$ \\
\hline ALMEIDA et al. ${ }^{21}$ & $\begin{array}{l}\text { Insvestigar os efeitos do ritmo } \\
\text { musical em aspectos fisiológicos, } \\
\text { afetivos e perceptuais, bem como o } \\
\text { desempenho do ritmo de caminhada } \\
\text { AS. }\end{array}$ & 28 mulheres & $\begin{array}{l}29-51 \\
\text { anos }\end{array}$ & Esteira & $\begin{array}{l}\mathrm{FC}, \mathrm{OMNI} \\
\mathrm{VO}_{2} \max \end{array}$ & $\begin{array}{l}\text { A música mais rápida promove uma } \\
\text { classificação mais elevada do esforço } \\
\text { percebido e maior desempenho no ritmo de } \\
\text { caminhada AS sem alterar significativamente } \\
\text { as variáveis fisiológicas ou a resposta afetiva. }\end{array}$ \\
\hline OREPIC et al. ${ }^{22}$ & $\begin{array}{l}\text { Determinar as respostas fisiológicas } \\
\text { reais durante o exercício de patinação } \\
\text { em ritmo AS de esforço em jovens e } \\
\text { comparar diferentes métodos para a } \\
\text { estimativa da intensidade do exercício } \\
\text { durante esta modalidade. }\end{array}$ & $\begin{array}{l}7 \text { homens } \\
10 \text { mulheres }\end{array}$ & $\begin{array}{l}29 \text { anos } \\
27 \text { anos }\end{array}$ & $\begin{array}{l}\text { Patinação em } \\
\text { asfalto }\end{array}$ & $\begin{array}{l}\mathrm{FC}, \mathrm{IPE} \\
\mathrm{VO}_{2} \max \\
\text { telemetria }\end{array}$ & $\begin{array}{l}\text { A patinação in-line recreativa em ritmos AS } \\
\text { induz respostas fisiológicas que são suficientes } \\
\text { para melhorar a aptidão cardiovascular em } \\
\text { adultos saudáveis. }\end{array}$ \\
\hline
\end{tabular}




\begin{tabular}{|c|c|c|c|c|c|c|}
\hline DOURIS et al. ${ }^{23}$ & $\begin{array}{l}\text { Comparar as respostas fisiológicas } \\
\text { e psicológicas do Nintendo Wii Fit } \\
\text { "Free Run" sob intensidade AS com } \\
\text { a realização de um padrão universal } \\
\text { para AF de intensidade moderada ( } 30 \\
\text { minutos de caminhada rápida). }\end{array}$ & $\begin{array}{l}9 \text { homens } \\
12 \text { mulheres }\end{array}$ & 23,2 anos & $\begin{array}{l}\text { Esteira e } \\
\text { Nintendo Wii } \\
\quad \text { Fit }\end{array}$ & $\begin{array}{l}\text { FC, IPE } \\
\text { Ao jogar o } \\
\text { Nintendo Wii } \\
\quad \text { Fit }\end{array}$ & $\begin{array}{l}\text { "Free Run" com uma intensidade AS, } \\
\text { estudantes universitários têm o potencial } \\
\text { de superar as intensidades de exercício } \\
\text { alcançadas ao executar um padrão } \\
\text { convencional para exercício de intensidade } \\
\text { moderada, como caminhada rápida em esteira. }\end{array}$ \\
\hline DA SILVA et al. ${ }^{24}$ & $\begin{array}{l}\text { Determinar se o ambiente (esteira e } \\
\text { campo) influencia diferencialmente } \\
\text { nas respostas fisiolpogicas, } \\
\text { perceptivas e afetivas ao exercício } \\
\text { com intensidade AS. }\end{array}$ & $\begin{array}{l}17 \text { homens } \\
17 \text { mulheres }\end{array}$ & $\begin{array}{c}24 \text { anos } \\
22,5 \text { anos }\end{array}$ & $\begin{array}{l}\text { Esteira e } \\
\text { campo }\end{array}$ & $\begin{array}{l}\mathrm{FC}, \mathrm{IPE}, \mathrm{VO}_{2} \\
\text { máx, VA }\end{array}$ & $\begin{array}{l}\text { As respostas fisiológicas, perceptivas e } \\
\text { afetivas foram influenciados pela configuração } \\
\text { ambiental, demonstrando ser mais agradável e } \\
\text { maior intensidade ao ar livre. }\end{array}$ \\
\hline $\begin{array}{l}\text { SIMPSON; MUNRO; } \\
\text { STEELE }{ }^{25}\end{array}$ & $\begin{array}{l}\text { Determinar como as variações na } \\
\text { carga afetaram a freqüência cardíaca, } \\
\text { postura e respostas subjetivas das } \\
\text { mulheres durante a caminhada } \\
\text { prolongada em trilha em ritmo AS, } \\
\text { para fornecer evidências de um } \\
\text { limite de massa de carga para essas } \\
\text { mulheres. }\end{array}$ & 15 mulheres & 22,3 anos & Esteira & $\begin{array}{l}\mathrm{FC}, \mathrm{IPE} \\
\text { análise de } \\
\text { movimento }\end{array}$ & $\begin{array}{l}\text { Embora as cargas de } 20 \%, 30 \% \text { e } 40 \% \\
\text { do PC estejam associadas a aumentos } \\
\text { estatisticamente significativos no IPE e } \\
\text { desconforto, as participantes não perceberam } \\
\text { essas cargas como pesadas ou causando } \\
\text { desconforto indevido até que carregassem } \\
40 \% \text { do peso. Com base nas concluiu-se que } \\
\text { um limite de carga de } 30 \% \text { do PC deve ser } \\
\text { recomendado para mulheres que caminham } \\
\text { em trilhas sob ritmo AS. }\end{array}$ \\
\hline DA SILVA et al. ${ }^{6}$ & $\begin{array}{l}\text { Avaliar se a idade influencia } \\
\text { diferencialmente as respostas } \\
\text { fisiológicas, perceptivas e afetivas } \\
\text { em mulheres sedentárias durante a } \\
\text { caminhada em ritmo AS. }\end{array}$ & $\begin{array}{c}\mathrm{G} 1=22 \\
\text { sedentárias } \\
\text { entre } 20-25 \\
\text { anos } \\
\text { G2 = 22 } \\
\text { sedentárias } \\
\text { entre } 30-35 \\
\text { anos } \\
\text { G3 = 22 } \\
\text { sedentárias } \\
\text { entre 40-45 } \\
\text { anos }\end{array}$ & $\begin{array}{c}\mathrm{G} 1=22 \\
\text { anos } \\
\mathrm{G} 2=33 \\
\text { anos } \\
\mathrm{G} 3=42,2 \\
\text { anos }\end{array}$ & Esteira & $\begin{array}{l}\mathrm{FC}, \mathrm{IPE}, \mathrm{VA} \\
\mathrm{VO}_{2} \max \end{array}$ & $\begin{array}{c}\text { A idade não influenciou significativamente nas } \\
\text { respostas perceptivas e afetivas, apenas nas } \\
\text { respostas fisiológicas. }\end{array}$ \\
\hline
\end{tabular}




\begin{tabular}{|c|c|c|c|c|c|c|}
\hline $\begin{array}{c}\text { BUZZACHERA et } \\
\text { al. }^{26}\end{array}$ & $\begin{array}{l}\text { Comparar as respostas fisiológicas, } \\
\text { perceptuais e afetivas durante } \\
\text { caminhada em ritmo AS por mulheres } \\
\text { adultas de três diferentes faixas } \\
\text { etárias. }\end{array}$ & $\begin{array}{c}\mathrm{G} 1=22 \\
\text { sedentárias } \\
\text { entre } 20-25 \\
\text { anos } \\
\text { G2 = 22 } \\
\text { sedentárias } \\
\text { entre } 30-35 \\
\text { anos } \\
\text { G3 = 22 } \\
\text { sedentárias } \\
\text { entre 40-45 } \\
\text { anos }\end{array}$ & $\begin{array}{l}\mathrm{G} 1=22 \\
\text { anos } \\
\mathrm{G} 2=33 \\
\text { anos } \\
\mathrm{G} 3=42,2 \\
\text { anos }\end{array}$ & Esteira & $\begin{array}{l}\mathrm{FC}, \mathrm{IPE}, \mathrm{VA}, \\
\mathrm{VO}_{2} \max \end{array}$ & 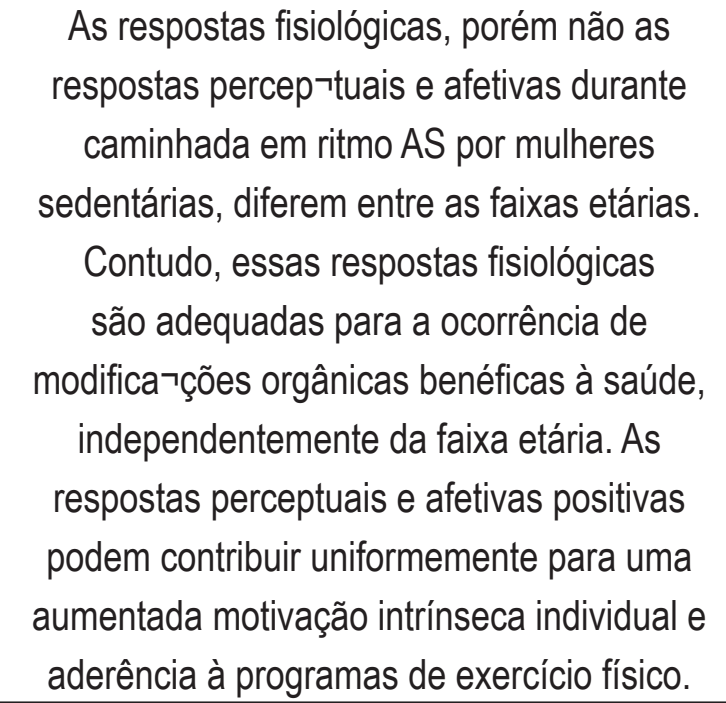 \\
\hline DA SILVA et al. ${ }^{27}$ & $\begin{array}{l}\text { Investigar a influência da adiposidade } \\
\text { nas respostas fisiológicas, perceptivas } \\
\text { e afetivas em mulheres sedentárias } \\
\text { durante a caminhada em ritmo AS. }\end{array}$ & $\begin{array}{c}\mathrm{G} 1=15 \\
\text { sedentárias com } \\
\text { baixo } \% \mathrm{GC} \\
\mathrm{G} 2=15 \\
\text { sedentárias com } \\
\text { médio } \% \mathrm{GC} \\
\mathrm{G} 3=15 \\
\text { sedentárias com } \\
\text { alto } \% \mathrm{GC} \\
\end{array}$ & $\begin{array}{l}\mathrm{G} 1=28,1 \\
\quad \text { anos } \\
\mathrm{G} 2=28,8 \\
\text { anos } \\
\mathrm{G} 3=32,4 \\
\text { anos }\end{array}$ & Esteira & $\begin{array}{c}\mathrm{FC}, \mathrm{IPE}, \mathrm{VA}, \\
\mathrm{VO}_{2} \max \end{array}$ & $\begin{array}{l}\text { As respostas fisiológicas tiveram diferença } \\
\text { significativa entre os grupos, mas não } \\
\text { obtiveram nas respostas afetivas. }\end{array}$ \\
\hline ELSANGEDY et al. ${ }^{28}$ & $\begin{array}{l}\text { Comparar as respostas fisiológicas } \\
\text { e perceptuais entre mulheres } \\
\text { com eutrofia, sobrepeso e obesas } \\
\text { durante a caminhada em ritmo } \\
\text { autosselecionado }\end{array}$ & $\begin{array}{c}\mathrm{G} 1=22 \\
\text { mulheres com } \\
\text { eutrofia } \\
\mathrm{G} 2=22 \\
\text { mulheres com } \\
\text { sobrepeso } \\
\mathrm{G} 3=22 \\
\text { mulheres } \\
\text { obesas }\end{array}$ & $\begin{array}{l}\mathrm{G} 1=30,8 \\
\text { anos } \\
\mathrm{G} 2=34,8 \\
\quad \text { anos } \\
\mathrm{G} 3=33,5 \\
\text { anos }\end{array}$ & Esteira & $\begin{array}{l}\mathrm{FC}, \mathrm{IPE}, \mathrm{VA}, \\
\mathrm{VO}_{2} \max \end{array}$ & $\begin{array}{l}\text { Os resultados do presente estudo demonstram } \\
\text { que mulheres obesas caminharam em } \\
\text { velocidade inferior a referida pelos outros dois } \\
\text { grupos. Contudo, respostas similares foram } \\
\text { registradas para os parâmetros fisiológicos } \\
\text { e para as respostas perceptuais. Esses } \\
\text { achados podem estar atrelados a ineficiência } \\
\text { biomecânica de caminhada encontrada em } \\
\text { sujeitos obesos durante a caminhada. }\end{array}$ \\
\hline
\end{tabular}




\begin{tabular}{|c|c|c|c|c|c|c|}
\hline HANSEN; SMITH ${ }^{11}$ & $\begin{array}{l}\text { Comparação do GE e da intensidade } \\
\text { AS entre a caminhada Nórdica } \\
\text { em aclive, plano e declive, com } \\
\text { diferentes comprimentos do bastão e } \\
\text { a caminhada comum. }\end{array}$ & $\begin{array}{l}1 \text { homem } \\
11 \text { mulheres }\end{array}$ & 50,6 anos & $\begin{array}{l}\text { Caminhada } \\
\text { Nórdica ao } \\
\text { ar livre e na } \\
\text { esteira. }\end{array}$ & $\begin{array}{l}\mathrm{FC}, \mathrm{GE} \\
\mathrm{VO}_{2} \max \end{array}$ & $\begin{array}{l}\text { Os praticantes de caminhada Nórdica } \\
\text { aumentaram seu GE em } 3 \% \text { durante a } \\
\text { caminhada nórdica em aclive, usando bastões } \\
\text { que eram } 7,5 \mathrm{~cm} \text { mais curtos em relação aos } \\
\text { que estavam acostumados. Notavelmente, } \\
\text { a maior taxa de gasto energético com polos } \\
\text { mais curtos foi obtida sem mudanças no } \\
\text { conforto auto-avaliado. Em comparação com a } \\
\text { caminhada normal, a taxa de gasto de energia } \\
\text { foi } 67 \% \text { maior durante a caminhada Nórdica, } \\
\text { embora seja similarmente confortável. }\end{array}$ \\
\hline RENDI et al. ${ }^{29}$ & $\begin{array}{l}\text { Determinar os efeitos psicológicos } \\
\text { agudos da corrida e ciclismo nas } \\
\text { cargas AS pelos participantes e } \\
\text { estabelecer a posteriori se a carga de } \\
\text { trabalho AS está relacionada com a } \\
\text { magnitude dos benefícios psicológicos } \\
\text { positivos esperados do exercício. }\end{array}$ & $\begin{array}{l}76 \text { homens } \\
4 \text { mulheres }\end{array}$ & 35 anos & $\begin{array}{l}\text { Cicloergômetro } \\
\text { e esteira }\end{array}$ & $\begin{array}{c}\text { FC, GE, } \\
\text { escala de } \\
\text { Likert }\end{array}$ & $\begin{array}{l}\text { Os resultados sugeriram que nem a forma } \\
\text { e nem a intensidade do exercício aeróbico } \\
\text { AS afetam as respostas psicológicas agudas } \\
\text { medidas. }\end{array}$ \\
\hline DA SILVA et al. $^{3}$ & $\begin{array}{c}\text { Investigar respostas perceptuais } \\
\text { e afetivas associadas ao ponto } \\
\text { de transição aeróbico-anaeróbico } \\
\text { e verificar se estas apresentam } \\
\text { diferenças em relação às respostas } \\
\text { afetivas durante caminhada em ritmo } \\
\text { AS. }\end{array}$ & $\begin{array}{l}45 \text { mulheres } \\
\text { sedentárias }\end{array}$ & 32,3 anos & Esteira & $\begin{array}{l}\mathrm{FC}, \mathrm{IPE}, \mathrm{VA}, \\
\mathrm{VO}_{2} \max \end{array}$ & $\begin{array}{c}\text { Escores do IPE e VA no LV foram similares aos } \\
\text { obtidos na caminhada em ritmo AS. }\end{array}$ \\
\hline
\end{tabular}

AF: atividade física; AS: autosselecionado; EA: escala afetiva; EEG: eletroencefalograma; FAS: Felt Arousal Scale; FC: frequência cardíaca; GE: gasto energético; GPS: Global Positioning System; IMC: índice de massa corporal; IPE: índice de percepção do esforço; LV: limiar ventilatório; PC: peso corporal; VA: valência afetiva; VO2max: volume máximo de oxigênio. 


\section{DISCUSSÃO}

O presente estudo apontou a FC e a PSE como as formas mais habituais de registro da intensidade durante os exercícios físicos. Além de serem métodos eficazes no controle da intensidade ${ }^{7}$, são de fácil manuseio e mensuração. Verificou-se também que atividades realizadas ao ar livre, como caminhada e corrida apresentaram resultados com melhores índices de sensações de prazer e bem-estar ${ }^{6,15,21,24,26-28}$. Tais exercícios são de fácil execução e podem ser realizados pela maioria das pessoas, fazendo com que estudos futuros possam reafirmar o aumento da aderência a longo prazo.

\section{Características dos praticantes, tipos de exercícios e formas de registro da intensidade.}

Dentre os vinte estudos selecionados para a presente revisão, dez deles foram realizados no Brasil, sendo sete no Estado do Paraná ${ }^{3,6,21,24,26-28}$, um no Estado do Rio de Janeiro ${ }^{17}$, um no Estado de Pernambuco ${ }^{15}$ e um no Estado do Rio Grande do Norte ${ }^{9}$. Os dez estudos restantes são internacionais, sendo quatro deles realizados nos Estados Unidos $^{16,18,19,23}$, três na Austrália ${ }^{10,20,25}$, um na Hungria ${ }^{29}$, um na Noruega ${ }^{11}$ e um na Croácia ${ }^{22}$. Esta diversidade é interessante pois apresenta aspectos transculturais que envolvem a prática de exercício físico autosselecionado em mulheres.

Quanto à idade das amostras dos estudos, é importante ressaltar que cinco estudos 6 ,19,20,22,23 dividiram as amostras em grupos por idade ou calcularam a média da idade de cada grupo separadamente. Sendo assim, observou-se que quatro grupos tinham idades entre 20 e 29 anos, seis com idades entre 30 e 39 anos e quatro com idades entre 40 e 49 anos. Dentre os demais estudos em que não houve divisão em grupos, foi possível averiguar que seis estudos foram realizados em mulheres com idades entre 20 e 29 anos ${ }^{16,17,22-25}$, quatro com idades entre 30 e 39 anos $^{3,9,10,29}$, três com idades entre 40 e 49 anos $^{15,18,21}$, um com idades entre 50 e 59 anos $^{11}$ e um com idades acima de 60 anos $^{20}$.

Sete estudos analisaram o percentual de gordura corporal (\%GC) para caracterizar as amostras, variando entre 14,4 e 43,05\% 3,10,15-17,22,24. É importante ressaltar que, para a análise dos resultados obtidos nos estudos em que haviam também sujeitos do sexo masculino, foram considerados apenas os dados das mulheres. Três estudos separaram amostra de acordo com o \%GC $9,27,28$, com o objetivo de classificar o nível de adiposidade (eutrófico, sobrepeso, obesidade), obtendo valores entre 20,8 e $34,7 \%$ de GC. De forma geral, os resultados apontaram que as mulheres obesas tendem a autosselecionar uma velocidade menor.

Três estudos separaram a amostra para avaliação do \%GC de acordo com a faixa etária, variando entre 25,1 a $31,7 \%$ de $\mathrm{GC}^{6,26,27}$. Foi observado que pessoas mais velhas realizaram um exercício na mesma velocidade que mulheres mais jovens, o que faz com que a intensidade relativa seja maior entre as mulheres mais velhas. 
Quanto ao efeito do exercício na massa corporal (MC), IMC e composição corporal um estudo analisou os efeitos do exercício em dois momentos (pré e pós) e descreveu os dados do nível de adiposidade apenas em kg (28,6 e $28,2 \mathrm{~kg}$, respectivamente) ${ }^{18}$, após um período de 4 semanas de treinamento, demonstrando diferença estatisticamente significativa. Dois estudos apresentaram o IMC como forma de classificar o grau de obesidade ${ }^{20,23}$, obtendo valores entre 23,7 e 24,1, e três estudos não forneceram nenhum tipo de dado que pudesse classificar de alguma forma o nível de adiposidade ${ }^{11,25,29}$. Através deste resultado, foi possível observar que dentre as classificações obtidas nos estudos, nenhum apresentou valores que levem a uma classificação abaixo do \% GC ideal.

Em relação aos tipos de exercícios encontrados nos estudos, cinco deles compararam a intensidade entre dois tipos de exercício, sendo dois deles em esteira e pista ${ }^{15,24}$, um em esteira e em simulação de jogo no Nintendo Wiir ${ }^{23}$, um em esteira e durante a caminhada Nórdica ${ }^{11}$ e um em esteira e cicloergômetro ${ }^{29}$. Dentre os estudos que analisaram a intensidade apenas em um exercício, onze deles utilizaram a esteira ${ }^{3,6,9,16,19-21,25-28}$, dois utilizaram o cicloergômetro ${ }^{10,17}$, um utilizou a patinação no asfalto22 e um utilizou Pedelec (bicicleta elétrica) ${ }^{18}$.

Os vinte estudos utilizaram a FC para mensurar a intensidade do exercício somados com outras formas de registro da mesma. Dentre elas, dezessete utilizaram a PSE (escala de Borg, OMNI, Likert) 3,6,9,10,15-17,20-29. Nove estudos utilizaram também diferentes escalas de sensações ${ }^{3,6,15-17,24,26-28}$, com o objetivo de analisar o nível de prazer durante o exercício. Além dessas formas de registro, todos os artigos desta revisão utilizaram outras formas de registro para identificar a carga de treino, estando de acordo com os objetivos de cada estudo ( $\mathrm{GE}, \mathrm{VO}_{2}$, distância percorrida).

\section{Comparação entre grupos com diferentes composições corporais}

Dentre os estudos que compararam grupos com diferente IMC ou \%GC, Elsangedy et al. ${ }^{9}$ verificaram o comportamento fisiológico e psicológico em mulheres com diferentes IMC e encontraram que, durante o teste máximo, mulheres obesas apresentaram menores respostas afetivas quando comparado à sessão com intensidade autosselecionada. Além disso, observou-se que o grupo de mulheres obesas autosselecionou velocidade menor em

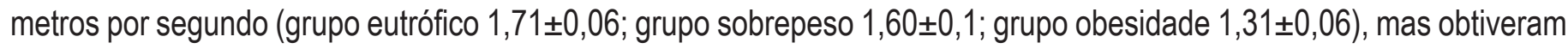
respostas fisiológicas similares aos grupos eutrófico e sobrepeso. Isso indica que mulheres obesas preferem não realizar exercícios com intensidade imposta quando comparado com intensidade autosselecionada e parece estar relacionado a má-adaptação biomecânica devido ao excesso de peso ${ }^{30}$.

Zdziarski et al. ${ }^{19}$ analisaram uma sessão única de exercício em corredores e corredoras eutróficas e com sobrepeso em que os voluntários corriam em uma velocidade autosselecionada durante 10 minutos, e posteriormente, corriam durante 1 minuto a uma intensidade imposta (maior que a autosselecionada). Os pesquisadores observaram 
que corredores e corredoras com sobrepeso autosselecionaram uma velocidade mais lenta, em quilômetros por hora $(8,5 \pm 1,3)$, e tiveram menor GE $(70,6 \pm 12,2)$ e maiores FC $(166 \pm 14)$ e repostas metabólicas durante a velocidade imposta quando comparado ao grupo eutrófico $(10,0 \pm 1,6 ; 54,6 \pm 10,4 ; 154 \pm 28$ respectivamente). Também não foram observadas diferenças significativas durante a intensidade autosselecionada. Embora o estudo não tenha analisado um grupo de obesos, é possível sugerir que o excesso de gordura corporal em pessoas com sobrepeso pode afetar na execução de uma corrida com intensidade imposta.

Já Da Silva et al. ${ }^{27}$ verificaram que a velocidade de caminhada autosselecionada não diferiu entre três grupos de acordo com $0 \% \mathrm{GC}$, enquanto as respostas do $\% \mathrm{VO}_{2}$ máx foram significativamente maiores no grupo de mulheres obesas (grupo eutrófico 52,8 $\pm 10,7$; grupo sobrepeso $55,8 \pm 6,8$; grupo obesidade $61,8 \pm 8,6$ ). Todos os três grupos autosselecionaram intensidades que se enquadram na faixa recomendada pelo $\mathrm{ACSM}^{4}$. Esses resultados podem indicar que, para realizar a mesma quantidade de trabalho, indivíduos com maior adiposidade utilizam maior porcentagem de sua potência aeróbica máxima ${ }^{31}$.

As respostas fisiológicas e perceptuais obtidas no estudo de Elsangedy et al. ${ }^{28}$ não apresentaram diferenças significativas entre os três grupos analisados de acordo com \%GC. No entanto, ao comparar a velocidade autosselecionada em metros por segundos, os grupos eutrófico $(1,69 \pm 0,11)$ e sobrepeso $(1,58 \pm 0,22)$ tiveram resultados maiores do que o grupo de mulheres obesas $(1,30 \pm 0,13)$. Essa variação na autosseleção da velocidade pode estar de acordo com as mudanças estruturais e funcionais causadas pelo excesso de peso corporal ${ }^{31}$.

É possível, assim, observar que a escolha da intensidade autosselecionada de exercício tende a estar na faixa recomendada do $\mathrm{ACSM}^{4}$, porém podendo ser realizada em menores intensidades quando os participantes possuem sobrepeso/obesidade. No entanto, Moura et al. ${ }^{7}$ encontraram que homens com sobrepeso tendem a autosselecionar uma intensidade acima do recomendado pelo $\mathrm{ACSM}^{4}$.

\section{Comparação entre grupos de diferentes faixas etárias}

Quando comparados grupos de diferentes faixas etárias, Da Silva et al. ${ }^{6}$ observaram que os três grupos etários analisados tiveram respostas afetivas positivas e autosselecionaram velocidades com valores semelhantes. $\mathrm{O} \% \mathrm{FC}$ máximo apresentou maior valor no grupo de mulheres entre 40-45 anos (grupo 20-25 anos 77,4 4 10,5; grupo 30-35 anos $77,8 \pm 7,1$; grupo $40-45$ anos $83,3 \pm 8,1$ ), permanecendo mais próximo ao limiar ventilatório (LV). No entanto, todos os grupos obtiveram respostas fisiológicas que se enquadraram nas recomendações do $\mathrm{ACSM}^{4}$, concluindo que não há influência da idade nas respostas afetivas, apenas nas respostas fisiológicas. 
Buzzachera et al. ${ }^{26}$ compararam as respostas entre diferentes grupos etários, observando que todos os grupos também autosselecionaram intensidades recomendadas pelo ACSM4, indicando, no entanto, que mulheres com maiores faixas etárias tendem a autosselecionar intensidades maiores de acordo com o \%FC máximo obtido (grupo 20-25 anos 73,2 $\pm 9,1$; grupo 30-35 anos 75,2 $\pm 7,1$; grupo $40-45$ anos $80,1 \pm 7,9$ ). Todos os grupos obtiveram respostas afetivas positivas e semelhantes, podendo contribuir para aumento da motivação individual.

Tais resultados reforçam a ideia de que pessoas mais velhas necessitam utilizar maior porcentagem de sua potência aeróbica máxima para realizar a mesma quantidade de trabalho de pessoas mais jovens, visto que a velocidade autosselecionada foi semelhante entre os grupos. No entanto, a intensidade autosselecionada de todos os grupos dos estudos citados acima estão dentro da faixa recomendada pelo ACSM4, além de apresentarem respostas afetivas positivas, que podem contribuir para maior motivação intrínseca durante o exercício ${ }^{32} \mathrm{e}$, consequentemente, aumentar a aderência.

\section{Comparação entre diferentes exercícios físicos}

Dentre os estudos que compararam duas formas diferentes de exercícios ou ambientes, Krinski et al. ${ }^{15}$ observaram que o ritmo (metros por segundo) autosselecionado foi ligeiramente mais elevado durante a caminhada

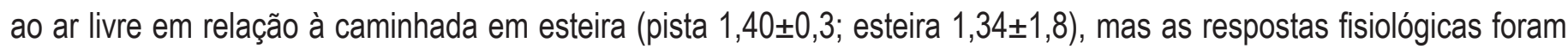

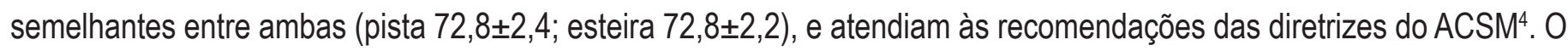
cenário ambiental influenciou as respostas psicológicas, demonstrando menor escore na escala de Borg ${ }^{8}$ e um estado afetivo agradável e mais favorável na condição externa. Esses dados podem levar a reflexão de que os indivíduos têm maior familiaridade ao caminhar no meio externo, pelo fato de estar mais próxima ao cotidiano, já que a esteira pode, em certos casos, produzir o temor de quedas. Mais importante ainda, caminhar ao ar livre parece ser mais agradável do que caminhar na esteira dentro de um laboratório.

Douris et al. ${ }^{23}$ comparou as respostas fisiológicas e psicológicas do Nintendo Wii Fit Free Run sob intensidade autosselecionada com a realização de caminhada rápida, tendo observado que a FC (Wii 142,4 $\pm 20,5$; esteira 123,2 $\pm 13,7$ ),

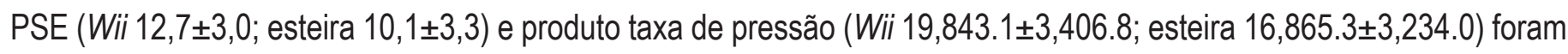
significativamente maiores durante o jogo de corrida livre no Nintendo Wii, estando de acordo com as recomendações do $\mathrm{ACSM}^{4}$. No entanto, as respostas afetivas tiveram menor escore no modo de jogo. Essas respostas afetivas podem estar relacionada com o fato de o indivíduo ter de realizar uma corrida estacionária durante todo o tempo no modo de jogo, além de necessitar de maiores demandas fisiológicas quando comparadas a uma caminhada rápida.

Glen et al. ${ }^{10}$ compararam a intensidade autosselecionada de um exercício em cicloergômetro sem modo de jogo (controle) e com dois diferentes modos de jogos de exergaming ativados. Os participantes eram previamente sedentários 
e sem experiência com exercícios em ciclo, e observou-se que os modos com distrações visuais tiveram uma PSE mais

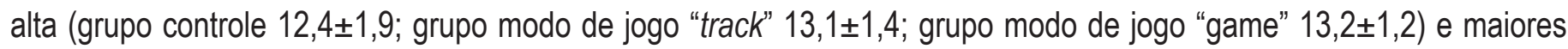
sensações de prazer. Nos dois modos de jogo, a intensidade permaneceu ligeiramente acima do LV e escala de afeto positivas. No entanto, o prazer diferiu significativamente entre o modo controle e os dois modos de jogo (grupo controle

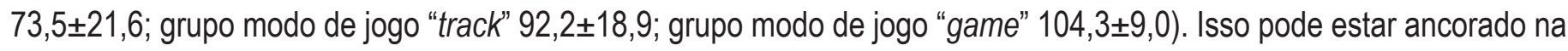
ideia de que quanto mais imersivo for o jogo, mais agradável será a experiência, sugerindo que esse tipo de atividade pode ser interessante para indivíduos sedentários e que estejam iniciando um programa de exercícios. Assim, o aspecto lúdico do jogo é um interessante estímulo para o exercício.

DaSilva et al. ${ }^{24}$ verificaram que indivíduos saudáveis estavam predispostos a autosselecionar menor velocidade de caminhada na esteira quando comparada à velocidade de caminhada em campo. No entanto, observaram-se maiores valores nas respostas fisiológicas e escore na escala de Borg ${ }^{8}$ na caminhada em esteira, além de uma valência afetiva menos positiva. A intensidade autosselecionada durante a caminhada em campo foi abaixo do recomendado pelo $\mathrm{ACSM}^{4}$ e significativamente abaixo do LV. Uma justificativa para esses resultados é que parecem estar relacionados com a idade, uma vez que uma meta-análise realizada por Ekkekakis ${ }^{33}$ argumenta que adultos jovens tendem a selecionar intensidades de exercício que estão abaixo da faixa recomendada pelo $\mathrm{ACSM}^{4}$, a menos que sejam instruídos para tal. Assim, partindo do ponto de vista prático, seria crucial a presença de um profissional para orientar esses indivíduos.

Ao comparar a caminhada com a caminhada Nórdica com diferentes inclinações, Hansen e Smith ${ }^{11}$ observaram que o GE foi $67 \%$ maior durante a caminhada Nórdica e 3\% maior durante 12 graus de subida quando comparada com 0 plano horizontal. Não foram observadas diferenças psicológicas e afetivas. Sendo assim, a caminhada Nórdica pode ser uma opção de combater a inatividade física das populações.

Rendi et al. ${ }^{29}$ compararam efeitos psicológicos e fisiológicos durante exercícios realizados na esteira e no cicloergômetro, sugerindo que nem a forma e nem a intensidade autosselecionada do exercício aeróbico afetam as respostas psicológicas agudas. Desta forma, sugere-se que ambos os exercícios podem promover respostas positivas e trazer benefícios na aptidão cardiorrespiratória, além de demonstrar que seria interessante que o indivíduo possa escolher o tipo de exercício de acordo com sua preferência.

\section{Outras condições de exercícios}

Orepic et al. ${ }^{22}$ determinaram as respostas fisiológicas obtidas durante a patinação em ritmo autosselecionado em jovens saudáveis e praticantes da modalidade e observaram que o exercício foi capaz de induzir respostas fisiológicas 
para promover melhorias na aptidão física, indicando que a modalidade induziu maior FC e PSE do que a esteira rolante, sendo categorizada como vigorosa, de acordo com o $\mathrm{ACSM}^{4}$. Essa intensidade alta pode estar relacionada com o fato de as mulheres avaliadas já serem praticantes da modalidade e terem um melhor condicionamento, o que pode ter ocasionado uma autosseleção de maiores intensidades.

Já Sherman et al. ${ }^{16}$ analisaram a intensidade de uma caminhada rápida com uma caminhada com intensidade similar a aula de Yoga mais intensa em adultas praticantes da modalidade. Quando comparada com a sessão de caminhada rápida, a resposta da FC foi significativamente menor, e foi acompanhado por um GE maior na caminhada em comparação com caminhada com intensidade da aula de Yoga, além de não ter sido observada diferença significativa na PSE. Com esses resultados, parece ser interessante combinar atividades como Yoga com outro tipo de exercício durante a semana, para acumular maior GE e atender às recomendações do ACSM ${ }^{4}$.

Smith et al. ${ }^{20}$ foram os únicos a analisarem o comportamento fisiológico de pessoas com idade acima de 60 anos sob intensidade autosselecionada. De forma geral, os idosos autosselecionaram intensidade acima do LV, estando próximo dele e mantendo um estado afetivo positivo. No entanto, essa condição pode elevar o risco, principalmente em indivíduos idosos com alguma comorbidade associada.

O único estudo que investigou os efeitos do ritmo musical sobre as respostas fisiológicas e psicológicas em mulheres que se exercitavam em intensidade autosselecionada foi o de Almeida et al. ${ }^{21}$. Dividiram a amostra em três grupos, sendo um controle (sem estimulação musical) e outros dois grupos com estilos musicais distintos. Observou-se que diferentes condições musicais não promoveram estímulos suficientes para alterar a intensidade do exercício, porém as respostas perceptivas foram maiores nos dois grupos que receberam estímulo musical. Desta forma, seria interessante a utilização de música durante a realização de uma atividade física, pois parece melhorar o bem-estar e, consequentemente, poderá aumentar a adesão ao exercício a longo prazo.

Simpson, Munro e Steele ${ }^{25}$ determinaram como as variações de carga de mochilas de mulheres que realizam caminhada em ritmo autosselecionado em trilha poderiam afetar a FC, postura e respostas subjetivas. As cargas foram de $0 \%, 20 \%, 30 \%$ e 40\% do PC. A medida que a carga aumentava, a FC não teve aumento concomitante. Observaram que a velocidade de caminhada é ajustada conforme a massa de carga é aumentada para minimizar o GE. O fato de a caminhada ter sido de forma autosselecionada pode explicar o fato de não ter variado a FC entre as cargas. Portanto, a caminhada em trilha não parece ser uma forma muito adequada quando existe uma carga muito alta nas mochilas, pois além de não ter variações na FC e velocidade (pelo fato de ajustarem essas variáveis para reduzirem o GE), podem trazer prejuízos para a postura, além de prejudicar articulações. 
DaSilva et al. ${ }^{3}$ investigaram respostas perceptuais e afetivas associadas ao ponto de transição aeróbicoanaeróbico. Os indivíduos eram previamente sedentários e os valores médios percentuais de $\mathrm{VO}_{2}$ e FC estiveram dentro dos padrões de intensidade estabelecidos pelo $\mathrm{ACSM}^{4}$ e próximos ao LV. Em relação à valência afetiva, não houve diferenças, indicando valores positivos em ambos. Sendo assim, os valores perceptuais e afetivos positivos de mulheres sedentárias em ritmo autosselecionado são similares e associados ao ponto de transição aeróbico-anaeróbico. Esses achados podem ser derivados do fato de que a realização de exercícios que estejam em uma intensidade acima do ponto de transição aeróbico-anaeróbico aumentam a demanda metabólica, contribuindo para uma maior PSE e menores sensações de prazer ${ }^{34}$, podendo reduzir a aderência ao exercício.

Lattari et al. ${ }^{17}$ compararam as respostas afetivas com a assimetria frontal obtida através do eletroencefalograma (EEG) induzida por sessões de exercícios prescritos e com intensidade autosselecionada. A amostra foi composta por jovens saudáveis e ativos pelo menos a 3 meses, e foram divididos em grupo controle, com intensidade autosselecionada e intensidade prescrita. Antes e depois de cada condição, foram registrados EEG, FC e escala de sensações. Não houve assimetria no lobo frontal após a condição do exercício autosselecionado, mas a mesma apresentou eficácia para produzir mudanças no condicionamento físico. As respostas afetivas positivas obtidas no estudo podem induzir a maior aderência a um programa de exercícios a longo prazo.

Petterman et al. ${ }^{18}$ verificaram a AF autosselecionada realizada por voluntários previamente sedentários adeptos ao Pedelec (Pedal Electric Cycle - bicicleta elétrica que sustenta o esforço do ciclista somente quando o mesmo estiver pedalando, através de sensores presentes no eixo dos pedais) e concluíram que o deslocamento diário por quatro semanas melhoraram significativamente alguns fatores de risco cardiometabólicos (melhora do $\mathrm{VO}_{2}$, potência máxima no teste, redução da PA, melhorias na tolerância da glicose). Além disso, os participantes cumpriram as recomendações da Organização Mundial da Saúde (OMS). Isso indica que o uso de Pedelec pode ser interessante para obter melhorias na aptidão física, uma vez que o equipamento pode ser utilizado como um veículo para deslocar-se para o trabalho, universidade, entre outros lugares.

Um dos fatores limitantes deste estudo foi a diversidade de atividades físicas encontrada nos artigos selecionados. Cada AF possui características específicas e respostas fisiológicas distintas, que podem influenciar na análise e comparação entre os resultados dos estudos. Além disso, outro limitador foi a heterogeneidade de faixa etária e condicionamento físico dos indivíduos dos estudos selecionados, talvez sendo necessária um maior refinamento dos critérios de inclusão. 


\section{CONSIDERAÇÕES FINAIS}

Foi observado que a maioria das mulheres com idade entre 20 e 39 anos, praticam exercícios aeróbicos que utilizam a intensidade autosselecionada; sendo as formas registro da intensidade mais habituais a FC e PSE.

Além disso, foi possível observar que atividades realizadas ao ar livre ou que possuem algum tipo de distração parecem trazer maiores sensações de prazer e bem-estar quando comparadas às atividades em laboratório. Em que os fatores que mais parecem influenciar na intensidade autosselecionada são o sobrepeso, devido a maior dificuldade de adaptação biomecânica causada, provavelmente, pelo excesso de peso; e a idade, sendo observado que pessoas mais velhas tendem a exercitar-se a uma intensidade relativa maior, utilizando mais de sua potência aeróbica máxima.

Por fim, esta revisão apontou evidências de que, quando a intensidade do exercício é autosselecionada, há maiores sensações de prazer e, consequentemente, um aumento da aderência a longo prazo. Além disso, muitos estudos demonstraram que a intensidade autosselecionada por diversos grupos atendem às recomendações promovendo melhorias no condicionamento físico e diminuindo o risco cardiovascular

\section{AGRADECIMENTOS}

Coordenação de Aperfeiçoamento de Pessoal de Nível Superior (CAPES).

\section{REFERÊNCIAS}

1. Ministério da Saúde. Vigitel Brasil 2016: Estimativas sobre frequência e distribuição sociodemográfica de fatores de risco e proteção para doenças crônicas nas capitais dos 26 Estados brasileiros e no Distrito Federal em 2016 [Internet]. Ministério da Saúde. 2017. 160p.

2. ABESO. Diretrizes brasileiras de obesidade 2016/ABESO. 4.ed - São Paulo, SP. 2016;1-188.

3. Silva S Da, Buzzachera CF, Elsangedy HM, Colombo H, Krinski K. Parâmetros perceptuais e afetivos como indicadores do ponto de transição aeróbico-anaeróbico na caminhada em ritmo auto-selecionado. Fit Perform J [Internet]. 2008;7(3):162-8.

4. Garber CE, Blissmer B, Deschenes MR, Franklin BA, Lamonte MJ, Lee IM, et al. Quantity and quality of exercise for developing and maintaining cardiorespiratory, musculoskeletal, and neuromotor fitness in apparently healthy adults: Guidance for prescribing exercise. Med Sci Sports Exerc. 2011;43(7):1334-59. 
5. Ministério do Esporte. Pesquisa Nacional por Amostra de Domicílios: Práticas de Esporte e Atividade Física. 2015.

6. Da Silva SG, Guidetti L, Buzzachera CF, Elsangedy HM, Colombo H, Krinski K, et al. Age and physiological, perceptual, and affective responses during walking at a self-selected pace. Percept Mot Skills [Internet]. 2010;111(3):963-78.

7. Moura BP De, Carlos J, Marins B, Roberto P. Autoselección de la velocidad de marcha de adultos con sobrepeso . ¿ Es suficiente la intensidad escogida para potenciar los beneficios de la salud ? Apunt Med E. 2011;46(169):11-5.

8. Borg GA. Psychophysical bases of perceived exertion. Med Sci Sports Exerc. 1982;14(5):377-81.

9. Elsangedy HM, Nascimento PHD, Machado DGS, Krinski K, Hardcastle SJ, DaSilva SG. Poorer positive affect in response to self-paced exercise among the obese. Physiol Behav [Internet]. Elsevier Inc; 2018;189:32-9.

10. Glen K, Eston R, Loetscher T, Parfitt G. Exergaming: Feels good despite working harder. PLoS One. $2017 ; 12(10): 1-12$.

11. Hansen EA, Smith G. Energy expenditure and comfort during nordic walking with different pole lengths. J Strength Cond Res. 2009;23(4):1187-94.

12. Krinski K, Elsangedy HM, Buzzachera CF, Colombo H, Alves RC, Santos B V., et al. Comparação das respostas fisiológicas e perceptuais obtidas durante caminhada na esteira em ritmo autosselecionado entre os sexos. Rev Bras Med do Esporte. 2010;16(4):291-4.

13. Downs S, Black N. The feasibility of creat- ing a checklist for the assessment of the methodological quality both of randomized and non-randomised studies of health care interventions. J Epidemiol Community Heal. 1998;52:377-384.

14. Rodrigues EQ. Aspectos metodológicos da aferição de atividade física em crianças de 7 a 10 anos de idade por meio do acelerômetro: Revisão sistemática da literatura. Universidade de São Paulo; 2013. 
15. Krinski K, Machado DGS, Lirani LS, DaSilva SG, Costa EC, Hardcastle SJ, et al. Let's Walk Outdoors! Self-Paced Walking Outdoors Improves Future Intention to Exercise in Women With Obesity. J Sport Exerc Psychol [Internet]. 2017;39(2):145-57.

16. Sherman SA, Rogers RJ, Davis KK, Minster RL, Creasy SA, Mullarkey NC, et al. Energy expenditure in vinyasa yoga versus walking. Int J Sport Nutr Exerc Metab. 2017;32:1-44.

17. Lattari E, Portugal E, Junior RSM, Oliveira BRR, Santos TM, Mura G, et al. Acute Affective Responses and Frontal Electroencephalographic Asymmetry to Prescribed and Self-selected Exercise. Clin Pract Epidemiol Ment Heal [Internet]. 2016;12(1):108-19.

18. Peterman JE, Morris KL, Kram R, Byrnes WC. Pedelecs as a physically active transportation mode. Eur J Appl Physiol. Springer Berlin Heidelberg; 2016;116(8):1565-73.

19. Zdziarski LA, Chen C, Horodysky M, Vincent KR, Vincent HK. Kinematic, Cardiopulmonary, and Metabolic Responses of Overweight Runners While Running at Self-Selected and Standardized Speeds. 2015;344(6188):1173-8.

20. Smith AE, Eston R, Tempest GD, Norton B, Parfitt G. Patterning of physiological and affective responses in older active adults during a maximal graded exercise test and self-selected exercise. Eur J Appl Physiol [Internet]. Springer Berlin Heidelberg; 2015;115(9):1855-66.

21. Almeida FAM, Nunes RFH, Ferreira S dos S, Krinski K, Elsangedy HM, Buzzachera CF, et al. Effects of musical tempo on physiological, affective, and perceptual variables and performance of self-selected walking pace. J Phys Ther Sci. 2015;27:1709-12.

22. Orepic P, Mikulic P, Soric M, Ruzic L, Markovic G. Acute physiological responses to recreational in-line skating in young adults. Eur J Sport Sci. 2014;14(SUPPL.1):37-41.

23. Douris PC, McDonald B, Vespi F, Kelley NC, Herman L. Comparison between nintendo Wii Fit aerobics and traditional aerobic exercise in sedentary young adults. J Strength Cond Res. 2012;1052-7. 
24. Silva SG Da, Guidetti L, Buzzavhera CF, Elsangedy HM, Krinski K, Campos W De, et al. Psychophysiological responses to self-paced treadmill and overground exercise. Sport Exerc. 2011;43(6):1002-9.

25. Simpson KM, Munro BJ, Steele JR. Effect of load mass on posture, heart rate and subjective responses of recreational female hikers to prolonged load carriage. Appl Ergon [Internet]. Elsevier Ltd; 2011;42(3):403-10. Available from: http://dx.doi.org/10.1016/j.apergo.2010.08.018

26. Buzzachera CF, Baldari C, Elsangedy HM, Krinski K, Santos B V, Campos W, et al. Comparação das respostas fisiológicas, perceptuais e afetivas durante a caminhada em ritmo autosselecionado por mulheres adultas de três diferentes faixas etárias. Rev Bras Med do Esporte [Internet]. 2010;16(5):329-34.

27. DaSilva SG, Guidetti L, Buzzachera CF, Elsangedy HM, Colombo H, Krinski K, et al. The influence of adiposity on physiological, perceptual, and affective responses during walking at a self-selected pace. Percept Mot Skills [Internet]. 2009;109(1):41-60.

28. Elsangedy HM, Krinski K, Buzzachera CF, Nunes RFH, Almeida FAM, Baldari C, et al. Respostas fisiológicas e perceptuais obtidas durante a caminhada em ritmo autosselecionado por mulheres com diferentes índices de massa corporal. Rev Bras Med do Esporte. 2009;15(4):287-90.

29. Rendi M, Szabo A, Szabó T, Velenczei A, Kovács Á. Acute psychological benefits of aerobic exercise: A field study into the effects of exercise characteristics. Psychol Health Med [Internet]. 2008;13(2):180-4.

30. Butterworth PA, Menz HB, Urquhart DM, Cicuttini FM, Pasco JA, Brennan SL, et al. The association between obesity and foot pain : metabolic, biomechanical or both? J Foot Ankle Res [Internet]. BioMed Central Ltd; 2015;8(Suppl 2):05.

31. Mattsson E, Larsson UE, Rössner S. Is walking for exercise too exhausting for obese women? Int J Obes. 1997;21:380-6.

32. Ekkekakis P, Hall EE, Petruzzello SJ. Variation and homogeneity in affective responses to physical activity of varying intensities : An alternative perspective on dose - response based on evolutionary considerations. (April 2015):37-41. 
33. Ekkekakis P. Let Them Roam Free ? Sport Med. 2009;39(10):857-88.

34. Ekkekakis P, Hall EE, Petruzzello SJ. Practical markers of the transition from aerobic to anaerobic metabolism during exercise: rationale and a case for affe 